\title{
Precipitation's complicated role in driving the abundance of an emerging disease vector in an urban, arid landscape
}

\author{
Erica Newman ${ }^{1}$, Xiao Feng ${ }^{2}$, Kathleen Walker ${ }^{3}$, Steven Young ${ }^{3}$, Kirk Smith $^{3}$, John \\ Townsend $^{3}$, Dan Damian ${ }^{3}$, José Soto ${ }^{3}$, and Kacey Ernst ${ }^{3}$ \\ ${ }^{1}$ University of Arizona \\ ${ }^{2}$ Florida State University \\ ${ }^{3}$ Affiliation not available
}

March 7, 2022

\begin{abstract}
Understanding drivers of disease vectors' population dynamics is a pressing challenge for human health, however, for short-lived organisms like mosquitoes, landscape-scale models must account for the highly local and rapid scale of their life cycle. Aedes aegypti, a vector of multiple emerging diseases, has been increasing in abundance in desert population centers, where water from precipitation could be a limiting factor. To explain this apparent paradox, we examined daily precipitation and Ae. aegypti abundances at >660 trapping locations per year for 3 years in the urbanized Maricopa County (metropolitan Phoenix), Arizona, USA. Through kriging of weather station data, we connected daily precipitation to subsequent trapped abundances of mosquitoes, and determined the timing and amount of precipitation that result in thresholds of interference with mosquito abundance. Large rainfall events resulted in no trapped mosquitoes 6-8 and 13-14 days later, while 10\% of all mosquitoes were trapped in long, precipitation-free periods.
\end{abstract}

\section{Introduction}

Predicting disease vector dynamics is of considerable importance for human and wildlife health globally, and is becoming increasingly imperative with global climate and land use changes (Lafferty 2009a, b; Alexander et al. 2018). Understanding the drivers of disease vectors' abundances and range expansions, as well as their life cycles, population structures, and interaction with human-modified environments will have immediate applications for prediction of disease exposure and transmission (Valenzuela-Sánchez et al. 2021), public health, and intervention efforts (Robbins \& Miller 2013; Cable et al. 2017; Little et al. 2017; Wimberly et al. 2020). However, one of the major challenges in predicting population-level dynamics of disease vectors is shared with that of global change ecology generally, that is, defining and incorporating the correct data to represent the relevant spatial and temporal extent of complex processes and ecological interactions (Wu \& David 2002; Anand et al. 2010; Newman et al. 2019).

In disease ecology, the mechanisms relating climate and weather to their effects on organisms at the appropriate scales are often poorly studied, and rely on lab-based studies rather than field-based ones (Caldwell et al. 2021). Relevant environmental conditions are often short-term, variable, and highly localized, and appropriate statistical approaches to connect local-scale weather information to organisms' life cycles may be lacking (Pascual \& Bouma 2009). Although laboratory experiments (Baker et al. 2000; Mordecai et al. 2019) can help define the causal relationships between weather variables and organismal biology, they do not translate directly to landscape-level or regional predictions. Many predictions of disease vector establishment and spread therefore rely on coarse-resolution climate predictors over broad extents, such as recent attempts 
employing climate envelope models (Khatchikian et al. 2011; Khormi \& Kumar 2014). These studies demonstrate that broad distributional patterns may be predictable with high error, as climate envelope models applied over large extents fail to match the resolution of fine-scale processes including weather changes, microclimates, and environmental variability related to structure, weather, and species interactions (Davis et al. 1998; Juliano 2009; Lembrechts et al. 2019; Caldwell et al. 2021), as well as reproductive success (Iwamura et al. 2020). Climate envelope approaches may be inappropriate for modeling emerging diseases when system-specific knowledge is ignored (Carlson et al. 2020; Valenzuela-Sánchez et al. 2021) or the goal is to predict population densities (Warren et al. 2020). Fine-scale environmental data is therefore necessary for testing hypotheses linking spatially- and temporally- structured population dynamics to underlying ecological variation (Albery et al. 2022).

The peridomestic mosquito Aedes aegypti (L.) (Diptera: Culicidae) is the primary vector of several major diseases, including the arboviruses dengue, Zika, chikungunya, and yellow fever. A highly invasive species originating from Africa, Ae. aegypti is now established throughout tropical and semitropical regions of the world (Christophers 1960; Tabachnick \& Powell 1979; Kraemer et al. 2015), and is expanding into the United States (Eisen \& Moore 2013; Ryan et al. 2019; Iwamura et al. 2020). As the species is strongly anthropophilic, its distribution is linked to urban environments and clustered human dwellings in rural areas (Soper 1967).

Although temperature responses and survival limits have been extensively studied for Ae. aegypti (e.g. BarZeev 1958; Reinhold et al. 2018), there may be other limits on the abundance of mosquitoes that depend on water availability. Less is known about the effects of environmental factors other than temperature on Ae. aegypti (Mordecai et al. 2019), such as seasonal and cumulative precipitation, and the importance of individual rainfall events on their choices of oviposition sites, larval development, and subsequent emergence. Precipitation is known to be an important factor at global, regional, and local scales, e.g., (Hopp \& Foley 2001; Wang et al. 2016; Benitez et al. 2021). However, it is still unknown if lack of precipitation can limit population density and overall abundance of Aedes mosquitoes, in part because water from anthropogenic sources can provide sufficient resources for container breeding mosquitoes (Trewin et al. 2013). Understanding the basic role of precipitation in driving mosquito abundance at fine scales can reveal where and when anthropogenic water sources become important.

\section{Life history of Aedes aegypti}

The life history and population biology of Ae. aegypti is dependent on water availability. Female mosquitoes lay their eggs in small water containers, and do not oviposit in large, permanent water bodies, irrigation ditches or temporary, shallow pools of water (Christophers 1960). This species has several traits that may result from adaptation to arid environments, for example, Ae. aegypti eggs can survive desiccation for months to years, and persistent water is not necessary (Faull \& Williams 2015; Mayilsamy 2019). Hatching of eggs is triggered by inundation by water. Progression through life stages for Ae. aegypti is temperature-dependent, requiring temperatures between $16^{\circ} \mathrm{C}$, and $34^{\circ} \mathrm{C}$ for successful development (Christophers 1960; Reinhold et al. 2018), with the transition from hatching to adult emergence occuring in as few as 7 days at higher temperatures (Couret \& Benedict 2014). Adults generally do not disperse beyond 30-60 m from their hatch site and tend to cluster around homes, but will rarely disperse as far as $500 \mathrm{~m}$ for oviposition sites (David et al. 2009; Brown et al. 2017; Marcantonio et al. 2019). The close association between Ae. aegypti and humans has allowed the species to establish in otherwise inhospitable climates, relying on human-created water sources such as stored drinking water for larval development (de Caires 1947; Focks et al. 1993; Barrera et al. 2011).

In Maricopa County, Arizona, USA (including metropolitan Phoenix), urbanization has led to human uses 
of water that create favorable conditions for mosquitoes. Anthropogenic uses of water have reduced the aridity of the local metropolitan area compared to the surrounding Sonoran Desert environment. Urban microclimates have altered temperature, humidity, and availability of oviposition sites, which constitute refugia for mosquito populations in desert cities. Ornamental planters in urban Arizona are known to be important breeding sites (Walker et al. 2018). While monitoring natural rainfall events is common, there are no comprehensive measurements of the large amounts of surface water generated by activities such as "urban greening": landscape maintenance, intentional flooding of lawns, and watering of ornamental plants; as well as recreational uses, ornamental features, car washing, and flooding fields for agriculture. Based on biological requirements, we expect that precipitation could be a limiting factor in mosquito activity. If lack of precipitation does not limit mosquito activity at trapping locations, we can conclude that mosquito breeding habitat is available from the only other source of water: anthropogenic uses.

\section{Precipitation and Aedes aegypti abundance}

A useful functional relationship has recently been proposed for regions that store drinking water, which specifies that increasing amounts of rainfall will have a complicated but deterministic, non-monotonic relationship with the abundance of Ae. aegypti (Shocket et al. 2020; Caldwell et al. 2021) (SI: Supplementary Background Material). Decreases in abundance are expected with increasing precipitation and less need for stored water (Trewin et al. 2013); increases with additional precipitation and habitat formation; and decreases with further precipitation flushing developing larvae out of containers, resulting in decreased abundance of adults (Seidahmed \& Eltahir 2016). This conceptual model provides important baseline expectations consistent with the biology of the container-breeding Ae. aegypti, but does not distinguish between precipitation amounts from individual rainfall events, and total accumulated precipitation prior to mosquito emergence.

We address how daily and cumulative precipitation affect mosquito abundance where piped water is available, and hypothesize that Ae. aegypti abundance and activity are strongly influenced by daily precipitation. Ae. aegypti may increase their activity (measured trap counts, and number of traps with at least one female in them) immediately following rainfall, as females may be selecting oviposition sites in those conditions. From 7-15 days following rainfall, counts may increase due to the development of eggs into adult mosquitoes. Alternatively, if mosquito abundance is constant after rainfall, this would imply that available oviposition sites are present at all times and are not a limiting factor. Ae. aegypti numbers may even decrease 5-20 days following rainfall if large amounts of rain flush away developing larvae from water-filled containers that contained immature life stages prior to the rainfall event.

We investigate these hypotheses and several other relationships between precipitation and mosquito presence and abundance by matching daily precipitation at the trapping locations to numbers of female Ae. aegypti captured each week by the Maricopa County Environmental Services Vector Control Division. These data were collected from 2014-2016, from a network of over 660 weekly-sampled $\mathrm{CO}_{2}$-baited traps distributed throughout Maricopa County, with more locations added in each year. An application of kriging algorithms (Yang et al. 2015) to weather station data allows us to interpolate local conditions between measured points, generate daily precipitation data layers, and match the scale of this predictor variable to the life cycle of mosquitoes. We can then reconstruct the relationship between daily precipitation amounts and timing, and the eventual outcomes of trapping. With increased spatial and temporal resolution available from kriging, we can generate new insights into how mosquitoes are directly affected by precipitation at the sites where they develop, emerge, and breed.

\section{Materials and Methods}




\section{Study area}

The current range of Ae. aegypti now includes almost all populated areas in Maricopa County, overlapping with high and increasing human population density, and land use modifications arising from increasing urbanization and suburbanization. Maricopa County spans 9,200 square miles ( ${ }^{\sim} 23,830$ sq. km), and contains 27 cities and towns including the metropolitan Phoenix area, and all or part of five tribal nations' federally designated reservation lands. Maricopa County counts 4.485 million people as residents as of the 2019 census.

Maricopa County is located in the biogeographic region of the Sonoran Desert, which has five seasons (winter, spring, fore-summer occurring in May and June, summer monsoon in July and August, and fall), and experiences two seasons of rainfall (winter and summer monsoon). Climate normals for the area from 1981-2010 include a mean minimum temperature of the coldest month (December) of $44.8^{\circ} \mathrm{F}(7.1 \mathrm{C})$; mean maximum temperature of the warmest month (July) of $106.1^{\circ} \mathrm{F}(41.2 \mathrm{C}$ ); and average annual precipitation of 8.03 inches $(20.4 \mathrm{~cm})$, with $25 \%$ of the rainfall occurring in July and August, and approximately half of the precipitation arriving throughout December-March (retrieved from the NOAA National Center for Environmental Information via the National Weather Service Forecast Office website: https://w2.weather.gov/climate). Recent climate change (post-2010) is increasing the maximum temperatures of the warmest months, as well as the average temperature, and the number of days over $100^{\circ} \mathrm{F}$. High and low temperatures in this region are known to exceed laboratory-derived physiological limits for Ae. aegypti (Christophers 1960; Farnesi et al. 2009; Reinhold et al. 2018).

\section{Mosquito trapping protocols}

Mosquitoes were trapped by Maricopa Environmental Services-Vector Control Division at trapping sites established throughout the urban and suburban areas of Maricopa County. Traps were placed at a density of one trap per square mile ( ${ }^{1610}$ meters), while accommodating urban structures (Fig. 1). Additional traps were placed temporarily in response to complaints about mosquito densities and in areas with reported human arbovirus cases. Standard $\mathrm{CO}_{2}$-baited traps (Silver 2007) were established at 842 unique sites (Table 1). Trapping occurred once per week at each site throughout the year. Traps were hung $\sim 1 \mathrm{~m}$ from the ground and left overnight to collect mosquitoes, which were then identified to species in the Maricopa County Environmental Services Vector Control Division laboratory and sorted by sex (SI: Notes on Aedes Trapping).

\section{Environmental data}

Daily precipitation data were downloaded from the Flood Control District of Maricopa County (FCDMC) weather stations $(n=355)$ (at https://www.maricopa.gov/625/Rainfall-Data). While this is a high density of weather stations compared to other similar sized regions in the US, the weather stations are clustered together, and geographic coverage was not complete. Values for daily precipitation for areas not directly measured were therefore spatially interpolated between weather stations using kriging. Kriging is a standard algorithm used to predict values in regions where data has been collected from the surrounding regions (Yang et al. 2015), that is used to search out parameters associated with known covariance data, and apply this information to new or unknown regions. In this study, a kriging algorithm was trained on daily precipitation data using elevation as a covariate using R 'automap' package (Hiemstra \& Hiemstra 2013). We adopted an automatic kriging function where a variogram model was fit using predefined models (spherical, exponential, Gaussian, Matern) with the default settings. The initial sill was estimated as the mean of the maximum and median of the semi-variance. The initial range was defined as 0.1 times the diagonal of the bounding box of the data, and the initial nugget was defined as the minimum semivariance. Elevation data were downloaded from the USGS National Elevation Dataset (http://ned.usgs.gov) and resampled to the resolution of 309 by 371 meters. 
Kriging was carried out on daily precipitation data for all days from October 2012-September 2017, to create spatially resolved, daily rasters for the study region (Fig. 1) (Newman \& Feng 2021). A relevant subset of these rasters were then matched to each individual trapping event, from 1 and 20 days prior to the event, inclusive. Interpolated precipitation data was then extracted by location. Dispersal distances of Ae. aegypti are known to be much lower than the distances between traps in the network. Therefore, information derived from kriging should better represent the conditions relevant to life cycle events of the mosquitoes than regional averages for precipitation.

\section{Statistical analyses}

We limited statistical analyses to female Ae. aegypti mosquitoes only, as only females transmit arboviruses. Female abundance in each trap ("counts") is used as the proxy metric for the activity of female Ae. aegypti. We first investigated the spatial clustering of mosquito counts across the study area by calculating the Global Moran's I statistic on aggregated female counts at each trapping location over three years. Global Moran's $I$ tests whether or not events are clustered, dispersed, or random in space.

We examined trap counts over months, and expected higher counts in trapping events that occur during June-October, as average minimum monthly temperatures have historically fallen below $15^{\circ} \mathrm{C}$ outside this range. Temperatures of $12-15^{\circ} \mathrm{C}$ and below are known to negatively affect adult female mosquito survival, and severely limit successful oviposition (Rueda et al. 1990; Farnesi et al. 2009; Yang et al. 2009; Tesla et al. 2018). However, microclimates in Maricopa County (Hayden et al. 2010; Larson \& Perrings 2013) can mitigate non-ideal temperature and precipitation conditions for mosquito development and lead to low emergence numbers, and temperature normals are changing rapidly in this region. We therefore included trapping events from all months in our analyses.

We then analyzed multiple relationships between prior precipitation, the number and proportion of traps with at least one female in them, and the number of female Ae. aegypti in traps. Related to their life cycle, Ae. aegypti counts from 1-6 days after rainfall events should correspond to increased activity of adult females, whereas abundance from 7-15 days after rainfall events may additionally correspond to the emergence of new adults (Christophers 1960; Couret \& Benedict 2014). We tested the following hypotheses by matching trap locations to spatially interpolated precipitation data at those sites for 20 days, counting backwards from the trap collection date:

H1: Adult mosquito activity and emergence is limited by accumulated precipitation prior to and after the egg-laying period in the vicinity of the trapping location. Cumulative precipitation was calculated as the sum of the spatially interpolated precipitation at the trapping location over 20 days.

H2: Adult mosquito activity and emergence is limited by prior single rainfall events. To assess this, we associated all trapping events with daily precipitation data at the trapping location on each previous day leading up to the trap collection date.

A distinct functional relationship that emerges from the empirical relationships would support the hypothesis being tested, even if that relationship is complicated or multimodal. On the other hand, we would interpret no significant correlation and explanatory power of predictor variables with female counts as a lack of control 
on mosquito abundance resulting from precipitation (the alternative hypotheses in each case). We would then conclude that anthropogenic sources of water are releasing the mosquito species from the constraints of water available from precipitation in urban contexts. Background levels of mosquito emergence with little or no measurable precipitation in the preceding 20 days were used to quantify the importance of anthropogenic water sources in driving mosquito abundance (SI: Supplementary Methods). Traps will catch mosquitoes from newly emerged to long-lived adult females. These may live slightly longer than 20 days in the wet tropics (Hugo et al. 2010), but their lifespan in the Sonoran Desert is expected to be shorter due to drier conditions, and dependent on anthropogenic water for survival as well as breeding.

To test the correlation between cumulative precipitation and female counts, we calculated Pearson's $r$ on repeatedly subsampled data ( 10 samples of $n=1000$ observations, performed with replacement), with female counts transformed on the $\log _{10}$ scale. This test was then followed by a fit to a simple linear model to assess explanatory power of cumulative precipitation on logarithmically transformed trap counts. For daily precipitation, trap outcomes and their associated prior precipitation data were used to test the probability that the observed pattern is significantly different than the random baseline (see SI: Supplementary Methods).

Data were cleaned and analyzed in the $\mathrm{R}$ programming language (v.4.0) (R Core Team 2020) with the packages 'dplyr' (Wickham et al. 2021) and 'raster' (Hijmans \& van Etten 2016), and Global Moran's I was calculated using package 'ape' (v5.0) (Paradis \& Schliep 2019). Mapping of data was carried out with the package 'ggmap' (Kahle \& Wickham 2013).

\section{Results}

Mosquito trapping

Maricopa County Environmental Services Vector Control Division collected mosquitoes from $\mathrm{CO}_{2}$-baited traps weekly and BG Sentinel traps at a total of 842 unique locations from January 2014-December 2016. Median distances between traps were determined to be $0.825 \mathrm{mi}(1328 \mathrm{~m}$ ) (using R package 'raster'; (Hijmans \& van Etten 2016)). There were a total of 100,757 discrete trapping events, and 122,257 Ae. aegypti mosquitoes were collected. The majority of traps did not contain Ae. aegypti, but there were 15,882 unique trapping events with 1 or more females (Table 1).

Of the trapped Ae. aegypti mosquitoes, $68.5 \%$ or 83,749 were female. Female counts, a proxy measure for total Ae. aegypti activity, varied with seasons. The monsoon season July-September coincides with high temperatures, reducing the generation time for the Ae. aegypti mosquitoes and providing additional water sources for breeding and survival. Based on historical data, we expected higher trapped abundance counts in June-October, but found high mosquito abundance both within the monsoon season, and in the surrounding months (Fig. 2) including March-November.

\section{Statistical analyses}

Our first analyses focused on the spatial distribution of female counts at each trapping location, aggregated over 3 years. Across all locations, 25 traps collected greater than 500 females in total over that time period. We calculated the Global Moran's $I$ statistic to understand the clustering of events over the extent of the study area (Fig. 3). We found Global Moran's $I_{o b s}=0.040\left(s d=0.007 ; p=2.7 \times 10^{-8}\right)$, with an expected value of $I_{\text {exp }}=-0.001$. High-count Ae. aegypti trapping events are significantly highly clustered in space compared to a baseline of a random distribution. 
Our next analyses were aimed at gaining insight into how the life cycle of the mosquito is affected by availability of water from precipitation, through application of kriging to daily precipitation data (Yang et al. 2015), and by matching trap locations to spatially interpolated precipitation data for 20 days preceding trap collection. For hypothesis H1, which tests whether or not adult mosquito emergence is limited by cumulative precipitation both before and after the egg-laying period in the vicinity of the trapping location, we calculated accumulated precipitation at each trapping location (Fig. 4; counts including zeros in SI: Fig. S1.). Pearson's $r$ calculated on logarithmically transformed trap counts against cumulative precipitation reveals a weak positive correlation $(r(\mathrm{df}>500)=0.22)$ that is nevertheless statistically significant $(p<0.001)$, because the relationship is approximately linear. However, a linear model fitted to the data (intercept $=$ 0.340 ; slope $=0.123 ; p<10^{-15}$ in each case, artificially significant due to large sample size) has extremely low explanatory power (adjusted $R^{2}=0.05$ ).

With hypothesis H2, we tested whether or not adult mosquito emergence is limited by prior single rainfall events. For this, we reconstructed daily precipitation at each location on days preceding trap collection. With a priori knowledge of the life cycle of Ae. aegypti at higher environmental temperatures, we expected increases in counts on days 7-15 days, and decreases 5-20 days following single rainfall events, with female counts at 1-6 days after rainfall events corresponding to increased activity of adult females, and counts from 7-15 days after rainfall additionally corresponding to the emergence of new adults. We instead found a surprisingly highly structured, multimodal relationship with daily precipitation and positive traps (Fig. 5a; also see Fig. S2), that we analyzed against a randomized draw from precipitation values in the data set for significance (below). In contrast, average precipitation calculated at each location for 20 days did not vary greatly across days (Fig. $5 \mathbf{b})$ (variance $=6 \times 10^{-6}$ inches).

High amounts of precipitation ( $>2.5$ inches) could occur at any time leading up to trap collection because trap data was pooled across calendar dates, but no traps contained female Ae. aegypti when $>2.5$ inches of precipitation occurred on days 2, 6-8, and 13-14, and day 20 prior to trap collection. We calculated the probability of obtaining the observed precipitation associated with positive trap counts by random chance (SI: Supplementary Methods). For a single day with this threshold, the probability of observing this pattern randomly is $\mathrm{P}$ (no observations [?] $2.5 \mathrm{in})=2.4 \times 10^{-6}$. For six days (without ordering), the upper limit of the probability of this distribution occurring from random chance is $\mathrm{P}$ (no observations [?] 2.5 in) ${ }^{6}$ $=2.1 \times 10^{-34}$. We conclude that it is statistically extremely unlikely that the observed pattern is consistent with a random distribution.

To assess the potential importance of anthropogenic water sources on driving patterns of mosquito abundance and activity, we examined all traps with at least one positive female count ("positive traps"; 15,882 observations) and sorted outcomes to examine which of these were associated with little to no measurable precipitation prior to collection (likely lower than the inundation requirements for developing eggs in containers), and for which more precipitation did not affect the maximum count in the trap: $<0.01 \mathrm{in}$. $(26.9 \%$; $\max$ count $=175)$, and $<0.02$ inches precipitation $(27.6 \%$; $\max$ count $=175)$. These traps contain $8-10 \%$ of total trapped females. We therefore estimate a background population of $10 \%$ of Ae. aegypti that are not

tied to precipitation patterns. The largest count events (1200 and 2200 females), in contrast, were associated with 0.4 and 0.5 inches of precipitation in single rainfall events.

\section{Discussion}

Understanding conditions that lead to greater presence and abundances of Ae. aegypti is critical to control- 
ling the spread of arboviruses to humans. We examined how the amount of preceding precipitation interacts with the Ae. aegypti life cycle to affect the abundance of trapped female mosquitoes. We found that the active season of Ae. aegypti in urban Maricopa County, Arizona is increasing in duration compared to historical norms. Summer temperatures exceed laboratory survival tolerances for Ae. aegypti, and their increased abundance during these periods may point to dispersal to suitable microhabitats (Hayden et al. 2010). We also examined the clustering of the most trapped females over three years $(>500$ females at a single trap location), and found these were highly clustered, indicating where it could be productive to search for larval development sites and target control efforts. Here, the specific locations of large outbreaks might be associated with clogged drains of Homeowner Association (HOA) planned communities (K. Walker, unpublished data) serving as breeding habitat. The interaction between precipitation and human-built structures may indeed be driving the emergence of a major disease vector in this region (Dieguez Fernandez 2010).

We hypothesized, following (Shocket et al. 2020) and (Caldwell et al. 2021), that cumulative rainfall over the period of 20 days prior to trap collection should influence the abundance of trapped mosquitoes, and also tested whether or not daily precipitation leading up to trapping had any influence on trap outcomes. We examined precipitation data leading up to a positive trap event (and also graphed the influence of precipitation on the counts in those traps SI: Fig. S2-Fig. S3). We found that neither average nor cumulative precipitation over 20 days is highly explanatory of trapped abundances and positive traps, however, we did find a highly structured and non-random pattern of daily precipitation thresholds above 2.5 inches.

Where no counts were recorded when there were single events of above 2.5 inches of precipitation between 6-8 days preceding trap collection day, we conclude precipitation strongly interferes with oviposition and larval survival. We note that no emergence after $>2.5$ inches at days 13-14 and at 20 days corresponds to two and three complete development periods for Ae. aegypti. It is possible that the flushing away of larvae for a previous generation is still detectable by its effects on the abundance of the next generation. No counts were observed when precipitation exceeded 2.5-3 inches at 1 and 2 days before trap collection; this could be interference with adult eclosure and emergence, suppressed mosquito movement, or counting errors due to wet specimens (SI: Notes on Aedes Trapping).

Precipitation is not a good predictor for mosquito activity and trapped abundance at every scale. While Ae. aegypti numbers overall increase with cumulative precipitation, the correlation is weak, the explanatory power of a best-fit linear model is low, and the predicted mosquito counts range from about 3-4 mosquitoes over the range of precipitation values. Similarly, there is no strong and unidirectional association between individual rainfall event amounts and the number of trapped individuals that could predict mosquito counts at landscape scales. However, kriging reveals a strong influence of both the timing and amount of daily precipitation. Although we expected to find that low precipitation limits Ae. aegypti numbers, we instead found a clear pattern of suppression of abundance following large rainfall events at certain times in the life cycle of the mosquito. We measure hard thresholds of 2.5 inches precipitation on several of the days before emergence, beyond which all mosquito counts are suppressed, although not all of the mechanisms are apparent. Where patterns of Ae. aegypti emergence and capture are not affected by low precipitation (as is true for between $27-28 \%$ of positive traps and $~ 10 \%$ of the total number of trapped females in this study), we conclude that anthropogenic water sources provide the only breeding habitat for Ae. aegypti populations (David et al. 2009; Hayden et al. 2010; Barrera et al. 2011; Walker et al. 2018). However, the true dependency of the population on anthropogenic water for all reasons (microclimate modification, survival, breeding, etc.) is likely much higher than $10 \%$.

Along with precipitation, it is well established that both temperature and relative humidity affect larval 
development rate, adult survival and activity. We looked uniquely at precipitation and trap data that were pooled across all trapping dates, which blurs the effect of varying levels of temperature and humidity, while allowing the patterns of trapped abundance that result from precipitation to become more apparent. With respect to the patterns shown in Fig. 5a, we observe several "troughs," where mosquito activity is not seen in correspondence with high levels of precipitation. We would expect these troughs to shift to the right with increasing temperatures and faster development times (Farnesi et al. 2009; Couret \& Benedict 2014). Relative humidity might show similar trends due to increased movement and survival among eggs and adults (Rowley \& Graham 1968; Faull \& Williams 2015; Schmidt et al. 2018), but this relationship may be more complicated because of the interaction with mosquito fecundity (Costa et al. 2010).

If kriging methods were also applied to local-scale variations in temperature and humidity, along with their interaction with precipitation in the environment, we might gain new insight into what makes up the microclimates that drive establishment patterns and abundance of Aedes mosquitoes across very different climates (Lembrechts et al. 2019). In this way, the search for commonalities among regions from a "microclimate perspective" might resolve some of the issues surrounding how to accurately predict Aedes invasions into new regions including humid, tropical regions, and the urbanized Sonoran Desert. We have provided a proof-of-concept that kriging with weather station precipitation data can resolve certain questions about precipitation and the Ae. aegypti life cycle in the presence of anthropogenic water sources, and outside of a laboratory setting. Kriging with multiple variables could bring "big data" science (and the computational storage and power required) to bear on questions of disease vector expansion, which has extreme significance to global human health. Broader applications of kriging with multiple variables could serve as a useful decision support tool for disease vector control in urban settings, as microclimates can be modified, unlike climate normals.

Beyond understanding the dynamics of disease vector populations and disease ecology applications, the application of kriging in conjunction with organismal life cycles will be useful in the context of conservation planning (Kusch \& Davy 2022). The ability to examine highly localized and variable conditions that precede ecological events and the distributions of seasonal phenological phenomena (Newman et al. 2021; Park et al. 2021) will have broad applicability across ecological questions and ecosystems. Kriging may become a valuable planning tool for many organismal-environment problems, including predicting outbreaks of tree-killing beetles (Gougherty \& Davies 2021), understanding drivers of arthropod declines (Dornelas \& Daskalova 2020), anticipating changes to water bodies after precipitation (Haines 1981; Lipp et al. 2001); and making more granular predictions of effects of global change on organisms such as amphibians (Walls et al. 2013; Catenazzi 2015), those dependent on specialized interaction partners (Kearns et al. 1998; Kiers et al. 2010), and rare and biogeographically limited species (Lyons et al. 2005; Enquist et al. 2019).

\section{Acknowledgements}

We thank the following people for feedback and edits to the manuscript: D. Hembry, R. Burger, A. Lien, B. Enquist. EAN and XF were supported by the Bridging Biodiversity and Conservation Science program at the University of Arizona.

\section{References}

Albery, G.F., Sweeny, A.R., Becker, D.J. \& Bansal, S. (2022). Fine-scale spatial patterns of wildlife disease are common and understudied. Funct. Ecol., 36, 214-225.

Alexander, K.A., Carlson, C.J., Lewis, B.L., Getz, W.M., Marathe, M.V., Eubank, S.G., et al. (2018). The Ecology of Pathogen Spillover and Disease Emergence at the Human-Wildlife-Environment Interface. 
In: The Connections Between Ecology and Infectious Disease (ed. Hurst, C.J.). Springer International Publishing, Cham, pp. 267-298.

Anand, M., Gonzalez, A., Guichard, F., Kolasa, J. \& Parrott, L. (2010). Ecological Systems as Complex Systems: Challenges for an Emerging Science. Diversity , 2, 395-410.

Baker, R.H.A., Sansford, C.E., Jarvis, C.H., Cannon, R.J.C., MacLeod, A. \& Walters, K.F.A. (2000). The role of climatic mapping in predicting the potential geographical distribution of non-indigenous pests under current and future climates. Agric. Ecosyst. Environ., 82, 57-71.

Barrera, R., Amador, M. \& MacKay, A.J. (2011). Population dynamics of Aedes aegypti and dengue as influenced by weather and human behavior in San Juan, Puerto Rico. PLoS Negl. Trop. Dis., 5, e1378.

Bar-Zeev, M. (1958). The effect of temperature on the growth rate and survival of the immature stages of Aedes aegypti (L.). Bull. Entomol. Res., 49, 157-163.

Benitez, E.M., Estallo, E.L., Grech, M.G., Frias-Cespedes, M., Almiron, W.R., Robert, M.A., et al. (2021). Understanding the role of temporal variation of environmental variables in predicting Aedes aegypti oviposition activity in a temperate region of Argentina. Acta Trop., 216, 105744.

Brown, H.E., Cox, J., Comrie, A.C. \& Barrera, R. (2017). Habitat and Density of Oviposition Opportunity Influences Aedes aegypti (Diptera: Culicidae) Flight Distance. J. Med. Entomol., 54, 1385-1389.

Cable, J., Barber, I., Boag, B., Ellison, A.R., Morgan, E.R., Murray, K., et al. (2017). Global change, parasite transmission and disease control: lessons from ecology. Philos. Trans. R. Soc. Lond. B Biol. Sci., 372.

de Caires, P.F. (1947). Aedes aegypti control in the absence of a piped potable water supply. Am. J. Trop. Med. Hyg., 27, 733-743.

Caldwell, J.M., LaBeaud, A.D., Lambin, E.F., Stewart-Ibarra, A.M., Ndenga, B.A., Mutuku, F.M., et al. (2021). Climate predicts geographic and temporal variation in mosquito-borne disease dynamics on two continents. Nat. Commun., 12, 1233.

Carlson, C.J., Chipperfield, J.D., Benito, B.M., Telford, R.J. \& O'Hara, R.B. (2020). Species distribution models are inappropriate for COVID-19. Nat Ecol Evol, 4, 770-771.

Catenazzi, A. (2015). State of the World's Amphibians. Annu. Rev. Environ. Resour., 40, 91-119.

CDC. (2017). Surveillance and Control of Aedes aegypti and Aedes albopictus in the United States ( No. CS303153-A). Centers for Disease Control and Prevention.

Christophers, S.R. (1960). Aedes aegypti: the yellow fever mosquito. CUP Archive.

Costa, E.A.P. de A., Santos, E.M. de M., Correia, J.C. \& Albuquerque, C.M.R. de. (2010). Impact of small variations in temperature and humidity on the reproductive activity and survival of Aedes aegypti (Diptera, Culicidae). Rev. Bras. Entomol., 54, 488-493.

Couret, J. \& Benedict, M.Q. (2014). A meta-analysis of the factors influencing development rate variation in Aedes aegypti (Diptera: Culicidae). BMC Ecol., 14, 3.

David, M.R., Lourenco-de-Oliveira, R. \& Freitas, R.M. de. (2009). Container productivity, daily survival rates and dispersal of Aedes aegypti mosquitoes in a high income dengue epidemic neighbourhood of Rio de Janeiro: presumed influence of differential urban structure on mosquito biology. Mem. Inst. Oswaldo Cruz, 104, 927-932.

Davis, A.J., Jenkinson, L.S., Lawton, J.H., Shorrocks, B. \& Wood, S. (1998). Making mistakes when predicting shifts in species range in response to global warming. Nature, 391, 783-786. 
Dieguez Fernandez, L. (2010). Aedes (St.) aegypti en tanques bajos y sus implicaciones para el control del dengue en Camaguey. Revista Cubana de.

Dornelas, M. \& Daskalova, G.N. (2020). Nuanced changes in insect abundance. Science.

Eisen, L. \& Moore, C.G. (2013). Aedes (Stegomyia) aegypti in the continental United States: a vector at the cool margin of its geographic range. J. Med. Entomol., 50, 467-478.

Enquist, B.J., Feng, X., Boyle, B., Maitner, B., Newman, E.A., Jorgensen, P.M., et al. (2019). The commonness of rarity: Global and future distribution of rarity across land plants. Sci Adv, 5, eaaz0414.

Farnesi, L.C., Martins, A.J., Valle, D. \& Rezende, G.L. (2009). Embryonic development of Aedes aegypti (Diptera: Culicidae): influence of different constant temperatures. Mem. Inst. Oswaldo Cruz, 104, 124-126.

Faull, K.J. \& Williams, C.R. (2015). Intraspecific variation in desiccation survival time of Aedes aegypti (L.) mosquito eggs of Australian origin. J. Vector Ecol., 40, 292-300.

Focks, D.A., Haile, D.G., Daniels, E. \& Mount, G.A. (1993). Dynamic life table model for Aedes aegypti (diptera: Culicidae): simulation results and validation. J. Med. Entomol., 30, 1018-1028.

Gorsich, E.E., Beechler, B.R., van Bodegom, P.M., Govender, D., Guarido, M.M., Venter, M., et al. (2019). A comparative assessment of adult mosquito trapping methods to estimate spatial patterns of abundance and community composition in southern Africa. Parasit. Vectors, 12, 462.

Gougherty, A.V. \& Davies, T.J. (2021). Host phylogenetic diversity predicts the global extent and composition of tree pests. Ecol. Lett.

Haines, T.A. (1981). Acidic precipitation and its consequences for aquatic ecosystems: A review. Trans. Am. Fish. Soc., 110, 669-707.

Hayden, M.H., Uejio, C.K., Walker, K., Ramberg, F., Moreno, R., Rosales, C., et al. (2010). Microclimate and human factors in the divergent ecology of Aedes aegypti along the Arizona, U.S./Sonora, MX border. Ecohealth, 7, 64-77.

Hiemstra, P. \& Hiemstra, M.P. (2013). Package "automap." Compare, 105, 10.

Hijmans, R.J. \& van Etten, J. (2016). raster: Geographic data analysis and modeling. $R$ package version, 2 .

Hopp, M.J. \& Foley, J.A. (2001). Global-Scale Relationships between Climate and the Dengue Fever Vector, Aedes Aegypti. Clim. Change, 48, 441-463.

Hugo, L.E., Cook, P.E., Johnson, P.H., Rapley, L.P., Kay, B.H., Ryan, P.A., et al. (2010). Field validation of a transcriptional assay for the prediction of age of uncaged Aedes aegypti mosquitoes in Northern Australia. PLoS Negl. Trop. Dis., 4, e608.

Iwamura, T., Guzman-Holst, A. \& Murray, K.A. (2020). Accelerating invasion potential of disease vector Aedes aegypti under climate change. Nat. Commun., 11, 2130.

Juliano, S.A. (2009). Species interactions among larval mosquitoes: context dependence across habitat gradients. Annu. Rev. Entomol., 54, 37-56.

Kahle, D. \& Wickham, H. (2013). Ggmap: Spatial visualization with ggplot2. $R$ J., 5, 144.

Kearns, C.A., Inouye, D.W. \& Waser, N.M. (1998). ENDANGERED MUTUALISMS: The Conservation of Plant-Pollinator Interactions. Annu. Rev. Ecol. Syst., 29, 83-112.

Khatchikian, C., Sangermano, F., Kendell, D. \& Livdahl, T. (2011). Evaluation of species distribution model algorithms for fine-scale container-breeding mosquito risk prediction. Med. Vet. Entomol., 25, 268-275. 
Khormi, H.M. \& Kumar, L. (2014). Climate change and the potential global distribution of Aedes aegypti: spatial modelling using geographical information system and CLIMEX. Geospat. Health, 8, 405-415.

Kiers, T.E., Palmer, T.M., Ives, A.R., Bruno, J.F. \& Bronstein, J.L. (2010). Mutualisms in a changing world: an evolutionary perspective. Ecol. Lett., 13, 1459-1474.

Kraemer, M.U.G., Sinka, M.E., Duda, K.A., Mylne, A.Q.N., Shearer, F.M., Barker, C.M., et al. (2015). The global distribution of the arbovirus vectors Aedes aegypti and Ae. albopictus. Elife, 4, e08347.

Kusch, E. \& Davy, R. (2022). KrigR - a tool for downloading and statistically downscaling climate reanalysis data. Environ. Res. Lett., 17, 024005.

Lafferty, K.D. (2009a). Calling for an ecological approach to studying climate change and infectious diseases. Ecology.

Lafferty, K.D. (2009b). The ecology of climate change and infectious diseases. Ecology, 90, 888-900.

Larson, E.K. \& Perrings, C. (2013). The value of water-related amenities in an arid city: The case of the Phoenix metropolitan area. Landsc. Urban Plan., 109, 45-55.

Lembrechts, J.J., Nijs, I. \& Lenoir, J. (2019). Incorporating microclimate into species distribution models. Ecography , 42, 1267-1279.

Lipp, E.K., Kurz, R., Vincent, R., Rodriguez-Palacios, C., Farrah, S.R. \& Rose, J.B. (2001). The effects of seasonal variability and weather on microbial fecal pollution and Enteric pathogens in a subtropical estuary. Estuaries, 24, 266.

Little, E., Biehler, D., Leisnham, P.T., Jordan, R., Wilson, S. \& LaDeau, S.L. (2017). Socio-Ecological Mechanisms Supporting High Densities of Aedes albopictus (Diptera: Culicidae) in Baltimore, MD. J. Med. Entomol., 54, 1183-1192.

Lyons, K.G., Brigham, C.A., Traut, B.H. \& Schwartz, M.W. (2005). Rare species and ecosystem functioning. Conserv. Biol., 19, 1019-1024.

Marcantonio, M., Reyes, T. \& Barker, C.M. (2019). Quantifying Aedes aegypti dispersal in space and time: a modeling approach. Ecosphere, 10.

Mayilsamy, M. (2019). Extremely Long Viability of Aedes aegypti (Diptera: Culicidae) Eggs Stored Under Normal Room Condition. J. Med. Entomol., 56, 878-880.

Mordecai, E.A., Caldwell, J.M. \& Grossman, M.K. (2019). Thermal biology of mosquito-borne disease. Ecology.

Newman, E.A., Breckheimer, I.K. \& Park, D.S. (2021). Disentangling the effects of climate change, landscape heterogeneity, and scale on phenological metrics. Cold Spring Harbor Laboratory.

Newman, E.A. \& Feng, X. (2021). Maricopa County, AZ interpolated daily precipitation rasters.

Newman, E.A., Kennedy, M.C., Falk, D.A. \& McKenzie, D. (2019). Scaling and Complexity in Landscape Ecology. Frontiers in Ecology and Evolution, 7, 293.

Paradis, E. \& Schliep, K. (2019). ape 5.0: an environment for modern phylogenetics and evolutionary analyses in R. Bioinformatics, 35, 526-528.

Park, D.S., Newman, E.A. \& Breckheimer, I.K. (2021). Scale gaps in landscape phenology: challenges and opportunities. Trends Ecol. Evol., 36, 709-721.

Pascual, M. \& Bouma, M.J. (2009). Do rising temperatures matter? Ecology.

R Core Team. (2020). The R project in statistical computing. A language and environment for statistical computing. R Foundation for Statistical Computing, 1. 
Reinhold, J.M., Lazzari, C.R. \& Lahondere, C. (2018). Effects of the Environmental Temperature on Aedes aegypti and Aedes albopictus Mosquitoes: A Review. Insects, 9.

Robbins, P. \& Miller, J.C. (2013). 11 The mosquito state. Ecologies and Politics of Health, 41, 196.

Rowley, W.A. \& Graham, C.L. (1968). The effect of temperature and relative humidity on the flight performance of female Aedes aegypti. J. Insect Physiol., 14, 1251-1257.

Rueda, L.M., Patel, K.J., Axtell, R.C. \& Stinner, R.E. (1990). Temperature-dependent development and survival rates of Culex quinquefasciatus and Aedes aegypti (Diptera: Culicidae). J. Med. Entomol., 27, 892-898.

Ryan, S.J., Carlson, C.J., Mordecai, E.A. \& Johnson, L.R. (2019). Global expansion and redistribution of Aedes-borne virus transmission risk with climate change. PLoS Negl. Trop. Dis., 13, e0007213.

Schmidt, C.A., Comeau, G., Monaghan, A.J., Williamson, D.J. \& Ernst, K.C. (2018). Effects of desiccation stress on adult female longevity in Aedes aegypti and Ae. albopictus (Diptera: Culicidae): results of a systematic review and pooled survival analysis. Parasit. Vectors, 11, 267.

Seidahmed, O.M.E. \& Eltahir, E.A.B. (2016). A Sequence of Flushing and Drying of Breeding Habitats of Aedes aegypti (L.) Prior to the Low Dengue Season in Singapore. PLoS Negl. Trop. Dis., 10, e0004842.

Shocket, M.S., Anderson, C.B., Caldwell, J.M., Childs, M.L., Couper, L.I., Han, S., et al. (2020). Environmental Drivers of Vector-Borne Diseases. In: Population Biology of Vector-Borne Diseases (ed. John M. Drake, Michael Bonsall, and Michael Strand). pp. 85-118.

Silver, J.B. (2007). Mosquito Ecology: Field Sampling Methods. Springer Science \& Business Media.

Soper, F.L. (1967). Dynamics of Aedes aegypti distribution and density. Seasonal fluctuations in the Americas. Bull. World Health Organ., 36, 536-538.

Tabachnick, W.J. \& Powell, J.R. (1979). A world-wide survey of genetic variation in the yellow fever mosquito, Aedes aegypti. Genetics Research, 34, 215-229.

Tesla, B., Demakovsky, L.R., Mordecai, E.A., Ryan, S.J., Bonds, M.H., Ngonghala, C.N., et al. (2018). Temperature drives Zika virus transmission: evidence from empirical and mathematical models. Proceedings of the Royal Society B: Biological Sciences, 285, 20180795.

Trewin, B.J., Kay, B.H., Darbro, J.M. \& Hurst, T.P. (2013). Increased container-breeding mosquito risk owing to drought-induced changes in water harvesting and storage in Brisbane, Australia. Int. Health, 5, $251-258$.

Valenzuela-Sanchez, A., Wilber, M.Q., Canessa, S., Bacigalupe, L.D., Muths, E., Schmidt, B.R., et al. (2021). Why disease ecology needs life-history theory: a host perspective. Ecol. Lett., 24, 876-890.

Walker, K.R., Williamson, D., Carriere, Y., Reyes-Castro, P.A., Haenchen, S., Hayden, M.H., et al. (2018). Socioeconomic and Human Behavioral Factors Associated With Aedes aegypti (Diptera: Culicidae) Immature Habitat in Tucson, AZ. J. Med. Entomol., 55, 955-963.

Walls, S.C., Barichivich, W.J. \& Brown, M.E. (2013). Drought, deluge and declines: the impact of precipitation extremes on amphibians in a changing climate. Biology, 2, 399-418.

Wang, X., Tang, S. \& Cheke, R.A. (2016). A stage structured mosquito model incorporating effects of precipitation and daily temperature fluctuations. J. Theor. Biol., 411, 27-36.

Warren, D.L., Matzke, N.J. \& Iglesias, T.L. (2020). Evaluating presence-only species distribution models with discrimination accuracy is uninformative for many applications. J. Biogeogr., 47, 167-180.

Wickham, H., Francois, R., Henry, L. \& Muller, K. (2021). dplyr: A Grammar of Data Manipulation. R package version 1.0.7. https://CRAN.R-project.org/package=dplyr 
Wimberly, M.C., Davis, J.K., Evans, M.V., Hess, A., Newberry, P.M., Solano-Asamoah, N., et al. (2020). Land cover affects microclimate and temperature suitability for arbovirus transmission in an urban landscape. PLoS Negl. Trop. Dis., 14, e0008614.

Wu, J. \& David, J.L. (2002). A spatially explicit hierarchical approach to modeling complex ecological systems: theory and applications. Ecol. Modell., 153, 7-26.

Yang, H.M., Macoris, M., Galvani, K.C., Andrighetti, M.T.M. \& Wanderley, D.M.V. (2009). Assessing the effects of temperature on the population of Aedes aegypti, the vector of dengue. Epidemiology $\& 3$ Infection, $137,1188-1202$.

Yang, X., Xie, X., Liu, D.L., Ji, F. \& Wang, L. (2015). Spatial Interpolation of Daily Rainfall Data for Local Climate Impact Assessment over Greater Sydney Region. Advances in Meteorology, 2015.

\section{Figures and Table}

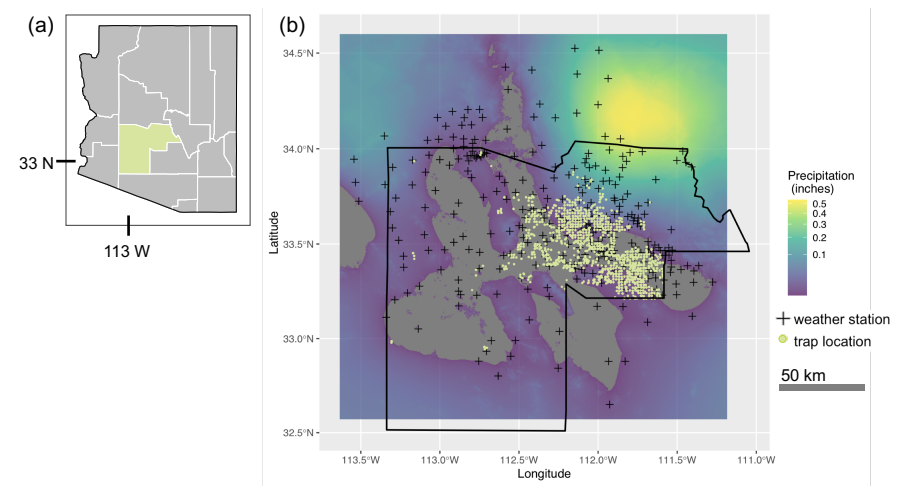

Figure 1: Example of daily precipitation (rainfall) data with spatial interpolation between 355 weather stations at the resolution of 10 arc-seconds ( $\sim 300$ meters). 842 traps were given spatially explicit, daily precipitation data from multiple rasters of interpolated precipitation. This allowed us to examine how the amount of precipitation received prior to trapping events interact to affect Ae. aegypti developmental period (i.e. the time from egg laying to emergence of the adult mosquito) and therefore the abundance of trapped adult mosquitoes. (a) A map of Arizona, USA is shown with Maricopa County highlighted. (b) In the map of Maricopa County, locations of weather stations are shown as black crosses, and trap locations are in light green; the kriged precipitation tile does not cover the entire extent of the county. 


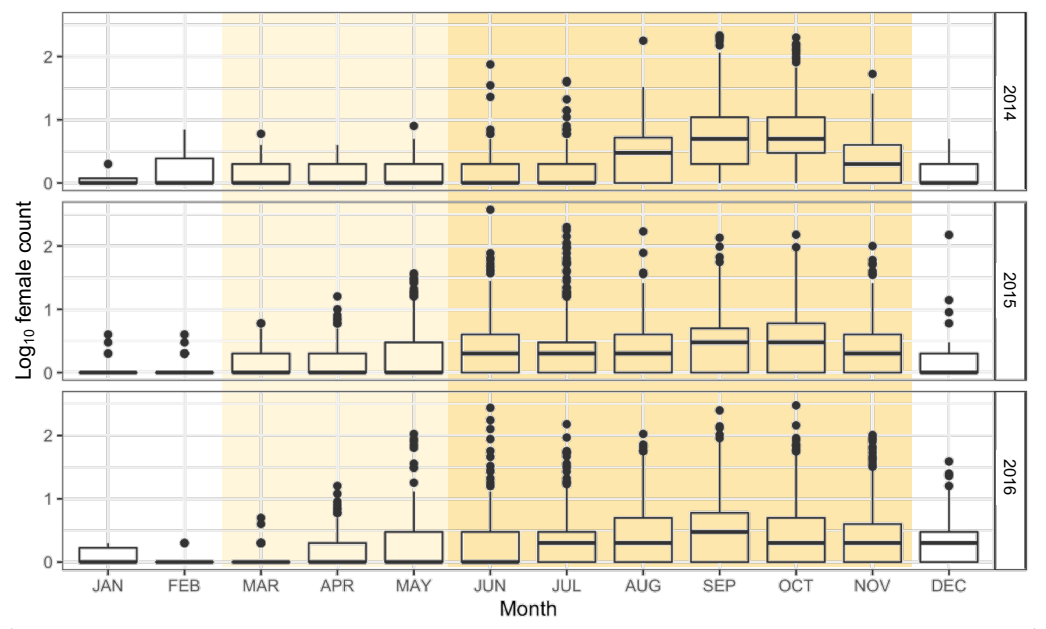

Figure 2: The number of trapped Ae. aegypti females is shown on a $\log _{10}$ scale by month for 2014-2016 (non-zero counts only). The yellow highlighted region represents the active season (March-November), and the "high activity" season (June-November) is shown in orange. These include all of the monsoon months (July-September). Historically, average minimum monthly temperatures are below the larval survival threshold for Ae. aegypti $\left(12^{\circ} \mathrm{C} / 54^{\circ} \mathrm{F}\right)$ in December-April (Farnesi et al. 2009), and exceed the laboratoryderived physiological limit for maximum temperature $\left(35^{\circ} \mathrm{C} / 95^{\circ} \mathrm{F}\right)$ in May-September (Reinhold et al. 2018), however, monthly temperature normals have been increasing in recent years. The activity period is therefore determined empirically.

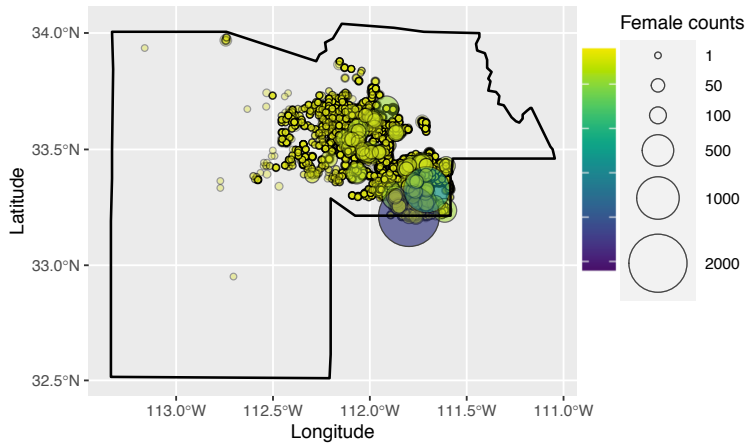

Figure 3: Spatial distribution of female Ae. aegypti counts recorded from 100,743 discrete trapping events at 842 locations from January 2014-December 2016 in Maricopa County, Arizona, USA. The observed Global Moran's $I_{o b s}=0.040\left(s d=0.007 ; p<0.001\right.$; expected value of $\left.I_{\text {exp }}=-0.001\right)$ for aggregated trapped female Ae. aegypti over three years (25 locations with $>500$ individual females) indicates that the locations with the highest number of are highly clustered in space compared to a random distribution baseline. A color ramp is provided to better visually distinguish between point sizes. 


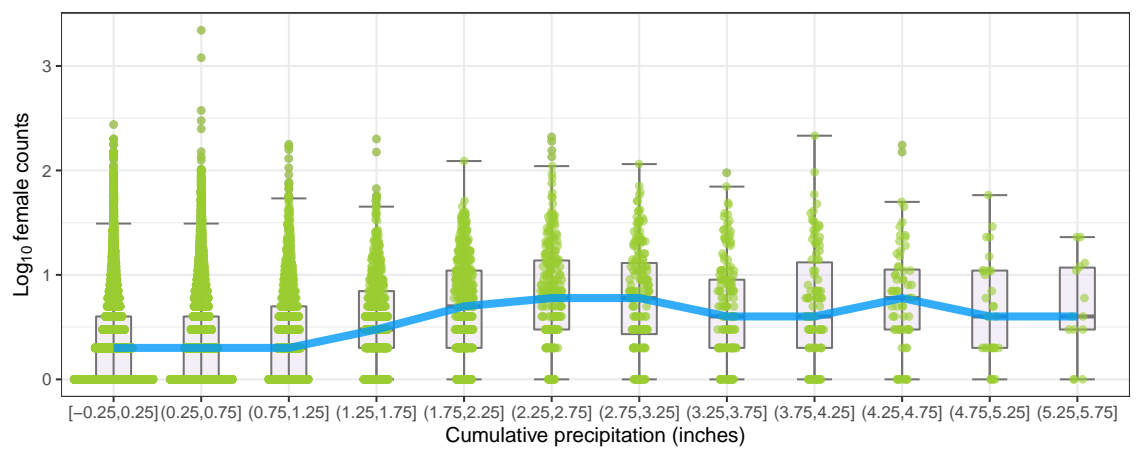

Fig. 4 All positive female counts from 15,882 unique trapping events plotted against cumulative precipitation received on days prior to trapping events. Female counts are shown on a $\log _{10}$ scale, and binned precipitation data represents cumulative precipitation (obtained from spatial interpolation) at each trapping location during the 20 days prior to the trap collection event. The blue line connects the median points of the boxplots. Pearson's correlation coefficient calculated on repeatedly subsampled data reveals a weak positive correlation between logarithmically transformed female counts and unbinned precipitation data $(r(\mathrm{df}>500)=0.22 ;)$ that is nevertheless statistically significant $(p<0.001)$, because the relationship is approximately linear. However, a linear model fitted to the data reveals the extremely low explanatory power of cumulative prediction on female counts (adjusted $R^{2}=0.05$ ). No distinct functional relationship is apparent. 

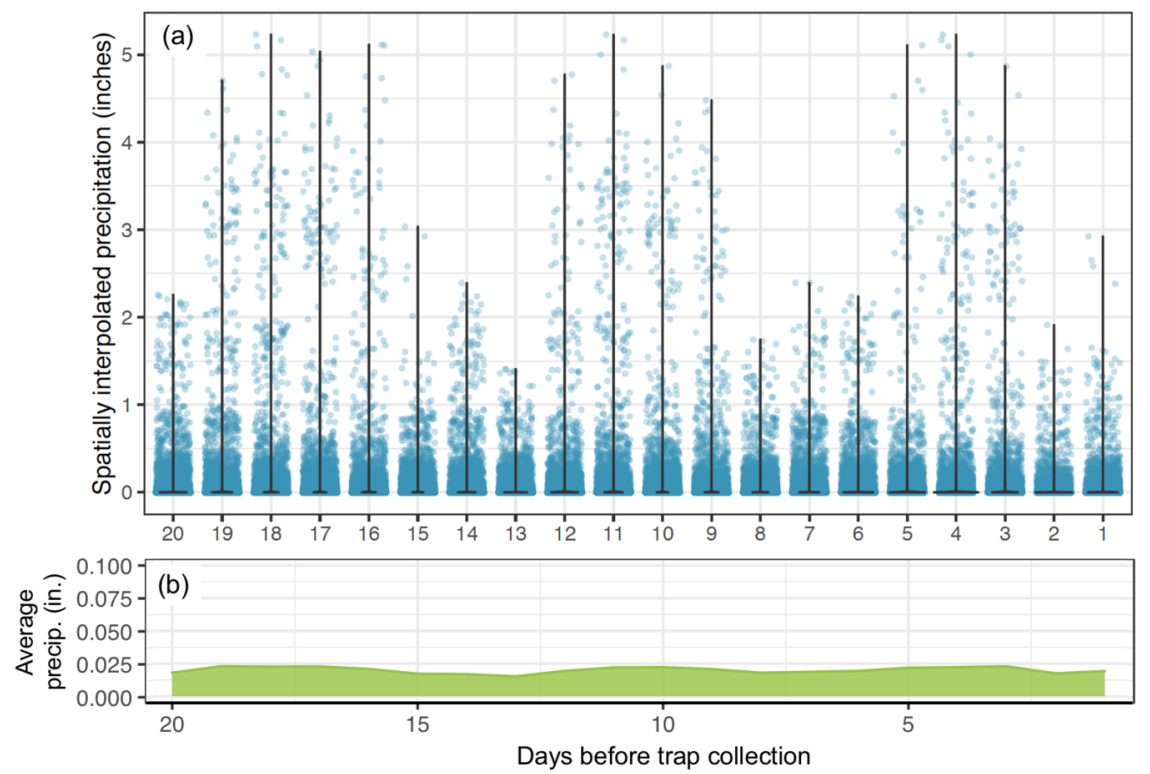

Figure 4: Figure 5 [formatting error]. Precipitation data on each day preceding trap collection. (a) All traps with positive counts of female Ae. aegypti are represented on each day $(n=15,822)$ as a blue point, mapping out the relationship between positive counts and 20 days of preceding precipitation data. High amounts of precipitation $(>2.5$ inches) could occur at any time leading up to trap collection, but no traps contained female Ae. aegypti when $>2.5$ inches of precipitation occurred on days 2, 6-8, and 13-14 prior to trap collection. Another threshold of $\sim 3$ inches of maximum precipitation is apparent for days 1 and 15 before trap collection. The calculated probability that this distribution is consistent with a random draw from available precipitation values is $<10^{-34}$. Precipitation data on days preceding trap collection is spatially interpolated from kriged rasters, and may overestimate actual precipitation. Dark lines are violin plots plotted over data points, with the vast majority of observations of precipitation equal to zero. (b) Average precipitation is low and nearly constant (>0.025 in., var [?]10-5 in.) across all observations at the trap locations.

Table 1. Summary of trapping effort, number of Aedes aegypti mosquitoes trapped, and number of and percentage of Ae. aegypti females from Maricopa County, Arizona, USA for 2014-2016. The number of traps with non-zero abundance are those that contained at least 1 male or female Ae. aegypti mosquito; those that only contained positive female counts are 15,882 traps, or $15.8 \%$ of total trap events.

\begin{tabular}{lllll}
\hline Year/Data & Trap type & Traps & Number of surveys & Number of traps with non-zero abundance (percen \\
$\mathbf{2 0 1 4}$ & $\mathrm{CO}_{2}$-baited & 666 & 28,131 & $3951(14.1 \%)$ \\
$\mathbf{2 0 1 5}$ & $\mathrm{CO}_{2}$-baited & 785 & 34,447 & $6051(17.6 \%)$ \\
$\mathbf{2 0 1 6}$ & $\mathrm{CO}_{2}$-baited & 794 & 37,901 & $6560(17.3 \%)$ \\
& BG Sentinel & 19 & 278 & $105(37.8 \%)$ \\
Totals & Unique traps: & 842 & 100,757 & $16,667(16.5 \%)$ \\
\hline
\end{tabular}


